\title{
Finite element simulations of viscoplastic flows in the presence of inertia and elastic effects
}

\author{
Fernanda B. Link, Sérgio Frey, \\ Departament of Mechanical Engineering, UFRGS, \\ 90050-170, Porto Alegre, RS \\ E-mail: feulink@mecanica.ufrgs.br, frey@mecanica.ufrgs.br, \\ Daniel D. dos Santos \\ Faculty of Mechanical Engineering, UFU, \\ 38408-144, Uberlândia, MG \\ E-mail: dallonder@femec.ufu.br, \\ Mônica F. Naccache, Paulo R. de Souza Mendes, \\ Departament of Mechanical Engineering, PUC-Rio, \\ 22453-900, Rio de Jaaneiro, RJ \\ E-mail: naccache@puc-rio.br, pmendes@puc-rio.br
}

\begin{abstract}
In this article some computations of inertia flows of elasto-viscoplastic materials down a one-to-four sudden expansion-contraction are performed. The phenomenon is model by the mass usual mass and and momentum balance equations together with an Oldroyd-type viscoelastic equation, modified to accommodate a dependence of both relaxation and retardation time as the viscoplastic viscosity on the material structure level. Such a model is approximated via a three-field Galerkin least-squares method in terms of extra-stress, velocity and pressure fields. According to its design, the compatibility conditions between the extra-stress-velocity and pressure-velocity finite element sub-spaces are bypassed, allowing the method uses equal-order finite element interpolations - more details see [8] and [1]. In the computations, the relevant adimensional elastic and viscous parameters are ranged in order to evaluate their influence on the elasto-viscoplastic fluid dynamics.
\end{abstract}

Keyword: Viscoplastic material, elasto-viscoplasticity, inertia flows, three-field GLS formulation.

\section{The motivation}

Non-Newtonian fluids flows are of vital relevance in many industrial processes. To cite only a few, we have polymeric flows in the plastic processing, drilling fluids in the oil industry, the manufacturing of shampoos and creams in the cosmetic business and the processing of milk and yogurts in the dairy industry.

Non-Newtonian flows through complex geometries are very usual in those processes as, for instance, significant changes of duct diameters and expansions and contractions of planar channels. Particularly, as viscoplastic fluids are involved[5], a given stress level - known as yield stress - needs to be achieved in order to the material to start to flow; otherwise, the material remains (almost) quiescent [2]. The former is called yielded regions and the latters is known as unyielded regions. The locus of points at stress equals the yield limit is denoted as yield surfaces. Some recent experiments have demonstrated that elasticity is present in unyielded 
region, playing an important role in the accurate determination of the morphology of unyielded regions.

The model employed in the present paper is suitable to apparent yield stress liquids, and is able to predict the elastic behavior often observed in these materials. In the present work we employ a novel elasto-viscoplastic constitutive model to predict the elastic effects in the unyielded regions of the flow through a planar abrupt expansion-contraction. To analyze the qualitative performance of the proposed model, the predicted results are compared with the experimental ones reported in [4] for asymmetric expansion-contractions.

\section{An elasto-viscoplastic model}

The non-thixotropic elasto-viscoplastic model used herein originates from the one proposed in [7] when the equilibrium time $t_{e q}$ equals zero, The equation for stress has the same form of the one for the Oldroyd-B model,

$$
\boldsymbol{\tau}+\theta_{1} \check{\boldsymbol{\tau}}=2 \eta_{e q}\left(\mathbf{D}+\theta_{2} \check{\mathbf{D}}\right)
$$

where the upper-convected time derivatives of $\boldsymbol{\tau}$ and $\dot{\gamma}$ are given by

$$
\check{\boldsymbol{\tau}} \equiv-\boldsymbol{\tau} \boldsymbol{u}-\nabla \boldsymbol{u}^{T} \boldsymbol{\tau} \quad \text { and } \quad \check{\mathbf{D}} \equiv-\dot{\gamma} \nabla \boldsymbol{u}-\nabla \boldsymbol{u}^{T} \dot{\gamma}
$$

The quantities $\theta_{1}$ and $\theta_{2}$ that appear in Eq. (1) are respectively the relaxation and retardation times. They are defined as

$$
\begin{aligned}
\theta_{1} & =\left(1-\frac{\eta_{\infty}}{\eta_{e q}}\right) \frac{\eta_{e q}}{G_{e q}} \\
\theta_{2} & =\left(1-\frac{\eta_{\infty}}{\eta_{e q}}\right) \frac{\eta_{e q}}{G_{e q}}
\end{aligned}
$$

The definitions of $\theta_{1}$ and $\theta_{2}$ above involve the equilibrium viscosity $\eta_{e q}$, the equilibrium elastic modulus $G_{e q}$, and the infinite-shear-rate viscosity $\eta_{\infty}$.

In the constitutive model, $\eta_{e q}$ and $G_{e q}$ (and hence $\theta_{1}$ and $\theta_{2}$ ) are assumed to be functions of the equilibrium structure parameter $\lambda_{e q}$, which is related to the equilibrium viscosity $\eta_{e q}(\dot{\gamma})$ (i.e. the flow curve of the material) through

$$
\lambda_{e q}(\dot{\gamma})=\frac{\ln \eta_{e q}(\dot{\gamma})-\ln \eta_{\infty}}{\ln \eta_{o}-\ln \eta_{\infty}}
$$

where $\eta_{0}$ is the zero-shear-rate viscosity and $\dot{\gamma} \equiv \sqrt{\frac{1}{2} \operatorname{tr} \dot{\gamma}^{2}}$ is the intensity of $\dot{\gamma}$. The equilibrium structure parameter $\lambda_{e q}$ is thus a scalar quantity that varies within the range $[0,1]$ and gives a measure of the structuring level of the microstructure, such that $\lambda_{e q}=0$ when the structuring level is minimum and $\lambda_{e q}=1$ when the material is fully structured. It is clear that this formulation assumes that there is a one-to-one relationship between the structuring level and the viscosity level. The equilibrium structure parameter $\lambda_{e q}$ can thus be seen as a normalized equilibrium viscosity function.

The dependence of the relaxation and retardation times on the structure parameter lends to the model a remarkable predictive capability, since in this way the predicted mechanical behavior ranges from the purely elastic up to the purely viscous behavior - see [7] for more details.

In this work, we employed the following expression for the equilibrium viscosity [3]:

$$
\eta_{e q}(\dot{\gamma})=\left[1-\exp \left(-\frac{\eta_{o} \dot{\gamma}}{\tau_{y}}\right)\right]\left\{\frac{\tau_{y}}{\dot{\gamma}}+K \dot{\gamma}^{n-1}\right\}+\eta_{\infty}
$$

In this equation, $\tau_{y}$ is the yield stress, $K$ the consistency index, and $n$ the power-law index. 
As discussed in previous publications [6],[7] and [8], three shear rates mark important transitions in the flow curve, namely $\dot{\gamma}_{o}, \dot{\gamma}_{1}$, and $\dot{\gamma}_{2}$. These shear rates are given by:

$$
\dot{\gamma}_{0}=\frac{\tau_{y}}{\eta_{0}}, \quad \dot{\gamma}_{1}=\left(\frac{\tau_{y}}{K}\right)^{1 / n}, \quad \dot{\gamma}_{2}=\left(\frac{\eta_{\infty}}{K}\right)^{1 / n-1}
$$

Following in [8], the dependence of the equilibrium elastic modulus $G_{e q}$ on the equilibrium structure parameter $\lambda_{e q}$ is given by:

$$
G_{e q}=G_{0} e^{m\left(\frac{1}{\lambda_{e q}}-1\right)}
$$

In this equation, $G_{0}$ is the structural elastic modulus of the fully structured material, and $m$ is a positive scalar parameter that dictates the sensitivity of $G_{e q}$ with $\lambda_{e q}$.

\section{Numerical Simulations}

Figure 1 shows a sketch of the analyzed geometry, a planar channel with a sudden expansion followed by a contraction. The employed boundary conditions to perform the numerical simulations are uniform parallel velocity $u_{0}$ at the channel inlet and outlet, no-slip and impermeability on channel walls and symmetry conditions along the channel centerline $\left(\partial_{x_{2}} u_{1}=u_{2}=\tau_{12}=0\right)$. The expansion-contraction aspect ratios on height $(H / h)$ and width $(L / h)$ are set as 6.3.

In order to guarantee fully-developed flow regions upstream and downstream channels, the mesh lengths either upstream or downstream of the expansion-contraction set equal to $20 h$. After a mesh independence procedure that compares the transverse dimensionless stress profile at the expansion-contraction center for each consecutive mesh refinement, the selected mesh, with 5, 200 bi-linear Lagrangian (Q1) finite elements, presents an overall error less then 1\% when compared to the next more refined mesh [9]. A detail of the central portion of the employed mesh is depicted on Fig. 2. It's important mentioning that in all simulations performed in this work, $\eta_{\infty} \dot{\gamma}_{1} / \tau_{0 d}=10^{-2}, \dot{\gamma}_{0 d} / \dot{\gamma}_{1}=10^{-4}, \tau_{0} / \tau_{0 d}=2$ and $m=2-[4]$.

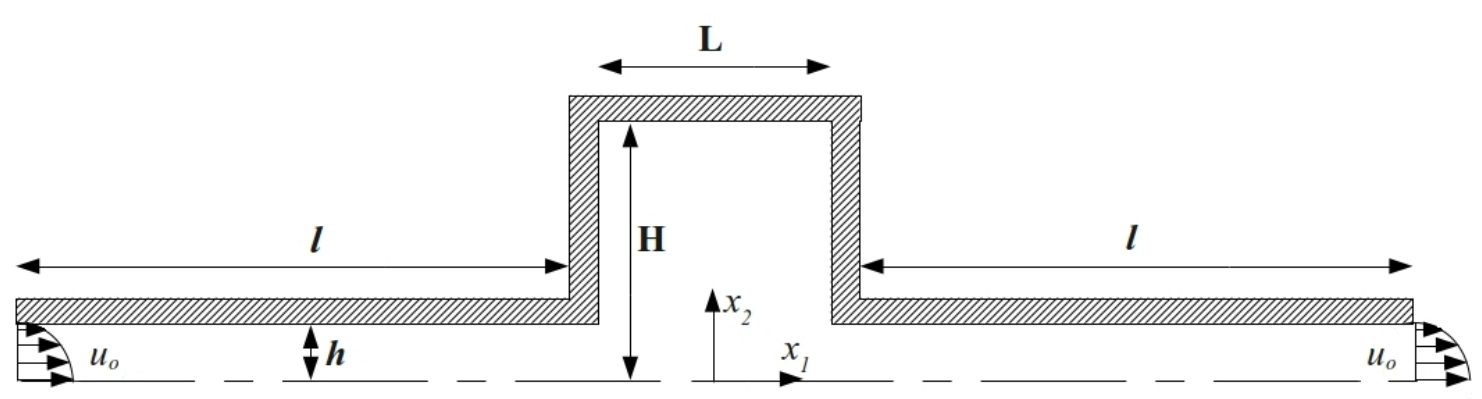

Figura 1: Geometry and boundary conditions.

An initial note must be made about the flow yield surfaces, which are usually defined as the surface where $\tau=\tau_{0}$. However, as $\dot{\gamma}$ varies some orders of magnitude with $\tau$ on the vicinity of $\tau=\tau_{0}$, this criterion may be inaccurate, once is susceptible to spurious oscillations. A more precise option is to defined the yield surfaces as the locus of points where $\dot{\gamma}=\dot{\gamma}_{0}$, with $\dot{\gamma}_{0}=\tau_{0} / \eta_{0}$ - see [7], for details.

The influence of the material elasticity in the morphology of the flow unyielded regions is shown in Fig. 3, for $\rho^{*}=0, J=5 \times 10^{3}, n=0.5$ and $U^{*}=0.1$, with $\theta_{0}^{*}$ equals to 0.75 and 25. For the higher value of the microstructure shear modulus $\left(\theta_{0}^{*}=25\right)$ the unyielded zones (the black ones in figures) are almost fore-aft symmetric in the absence of inertia and elasticity effects. For $\left(\theta_{0}^{*}=0.75\right)$, it is noted the tilting of the cavity unyielded region, what happens when the material is still highly structured and the stress is in the vicinity of the yield stress (highest possible stress while the material is highly structured). 


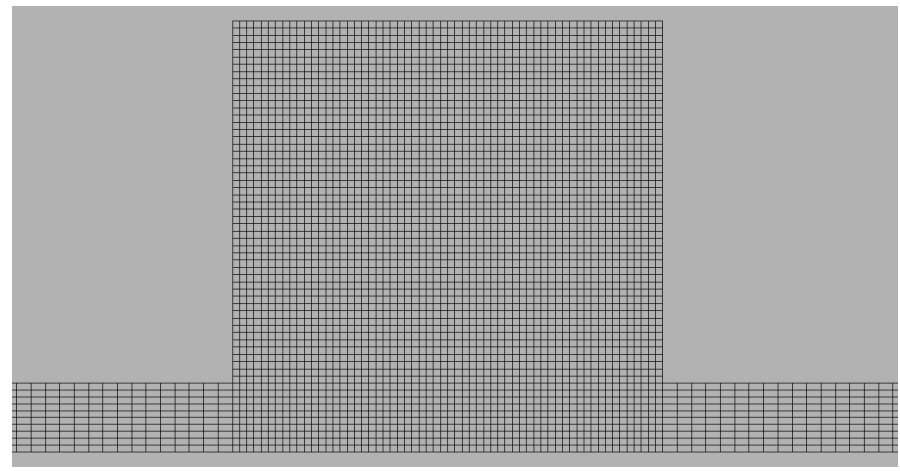

Figura 2: The central portion of the employed mesh.

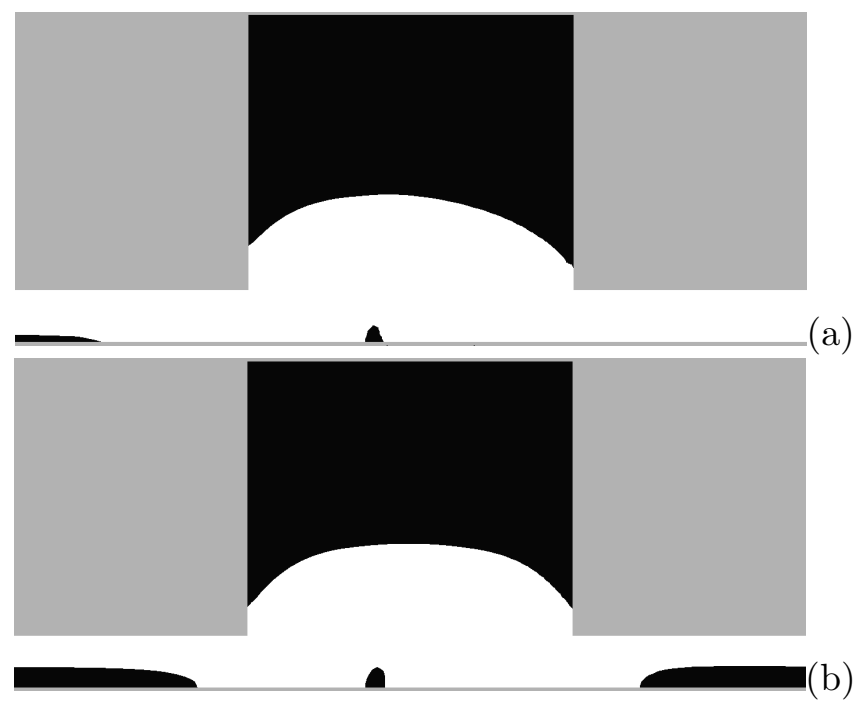

Figura 3: Effect of the elasticity on the flow, for $\rho^{*}=0, U^{*}=0.1, J=5 \times 10^{3}$ and $n=0.5-$ Yielded and unyielded regions for (a) $\theta_{0}^{*}=0.75$ and (b) $\theta_{0}^{*}=25$; elastic strain for (c) $\theta_{0}^{*}=0.75$ and (d) $\theta_{0}^{*}=25$.

Figure 4 show the effects of the dimensionless flow rate on the unyielded regions for $\rho^{*}=0$, $J=5 \times 10^{3}, n=0.5$ and $\theta_{0}^{*}=1.0$, with $U^{*}$ equals to 0.01 and 1.0. Large elastic strains occur throughout the flow domain for the smaller value of $U^{*}$, while are reduced for $U^{*}=1.0$. This behavior is observed due to the higher stress levels induced increasing the flow rate, which implies low structuring levels and hence high $G_{e q}$. The same reasoning can be used to explain the trend observed for the unyielded regions, decreasing monotonically as the dimensionless inlet velocity is increased.

The effect of inertia is illustrated on Fig. 5 for $\theta_{0}^{*}=1.0, J=5 \times 10^{3}, U^{*}=0.1$ and $n=0.5$, with $\rho^{*}=1.0$ and (b) $\rho^{*}=500$. The elastic strain field suffers minor effects of inertia once, for a fixed microstructure shear modulus, the dimensionless inlet velocity remains unchanged. Due to the type of scaling employed in this work [7], the changes on the flow field entailed by inertia are decoupled from the changes entailed by the imposed changes in the flow intensity. The shape of the cavity yield surface, however, changes significantly in the presence of inertial effects: while elasticity tends to tilt this yield surface counter-clockwise, inertia has the opposite effect. The cavity yield surface shown for $\rho^{*}=500$ is tilted clockwise, despite the high level of elasticity. 


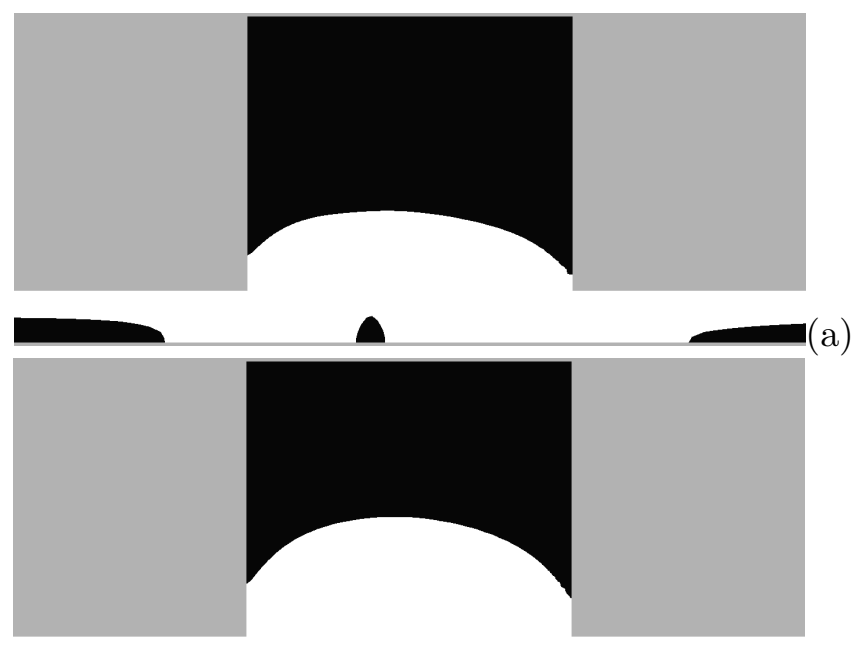

(b)

Figura 4: Effect of the dimensionless flow rate on the flow, for $\rho^{*}=0, \theta_{0}^{*}=1.0, J=5 \times 10^{3}$ and $n=0.5$ - Yielded and unyielded regions for (a) $U^{*}=0.01$ and (b) $U^{*}=1.0$; elastic strain for (c) $U^{*}=0.01$ and (d) $U^{*}=1.0$.

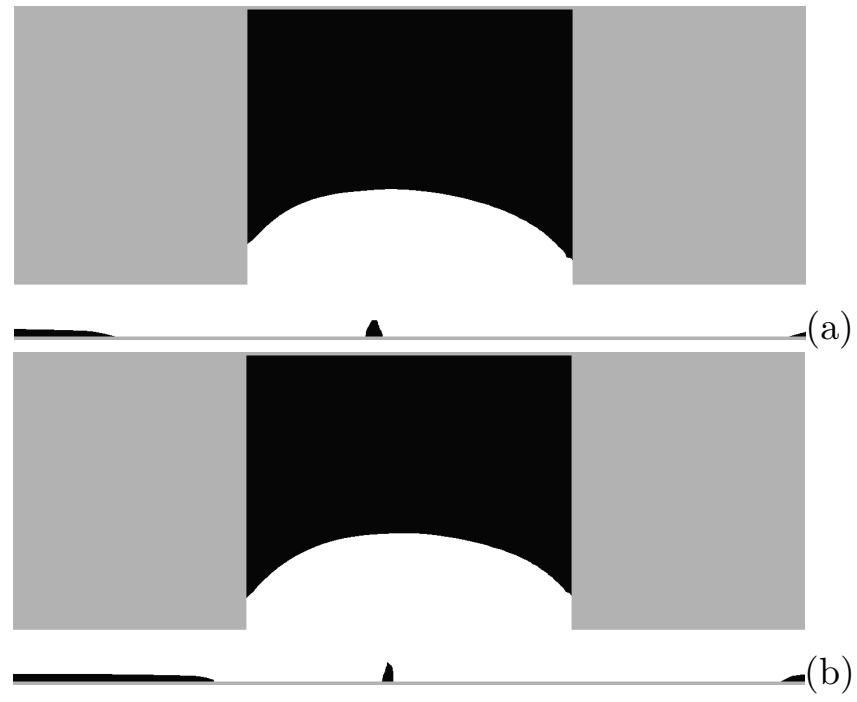

Figura 5: Effect of the inertia on the flow, for $\theta_{0}^{*}=1.0, J=5 \times 10^{3}, U^{*}=0.1$ and $n=0.5-$ Yielded and unyielded regions for (a) $\rho^{*}=1.0$ and (b) $\rho^{*}=500$; elastic strain for (c) $\rho^{*}=1.0$ and $(\mathrm{d}) \rho^{*}=500$.

\section{Final Comments}

Numerical finite element approximations for inertial flows of elasto-viscoplastic materials are herein performed. The mechanical modeling employed is made-up of the usual mass and momentum conservation for incompressible fluids, coupled with an Oldroyd-B-type equation and the the SMD viscoplastic function modified to be dependent of the flow strain rate. Such a model is approximated via a three-field Galerkin least-squares method in extra-stress, pressure and velocity.

Results intended to estimate the topology of yield surfaces as well as the determination of distribution of structuring level throughout the channel. The computational domain is partitioning into a bi-linear Lagrangian finite element mesh for all primal variables. The following non-dimension governing parameters are varied: the relaxation time, the flow intensity, the jump number, the power-law index and the equilibrium time. All simulations attest the relevance of 
the right determination of the structuring level on the accurate computation of the shape end location of unyielded regions, along with the strong influence of elasticity on the asymmetry of yield surfaces.

\section{Acknowledgements}

The authors acknowledge Petrobras S.A., MCTI/CNPq, CAPES, FAPERJ and FINEP for financial support.

\section{Reference list}

[1] Behr, A.M., Franca, L.P., Tezduyar, T.E., 1993. "Stabilized finite element methods for the velocity-pressure-stress formulation of incompressible flows", Computer Methods in Applied Mechanics and Engineering, vol. 104, pp. 31-48.

[2] Dos Santos, D.D,, Frey, S., Naccache, M.F., De Souza Mendes, P.R., 2011. "Viscosity Function for Yield-Stress Liquids", Applied Rheology, vol. 166, pp. 667-679.

[3] De Souza Mendes, P.R., Dutra, E.S.S., 2004. "Viscosity Function for Yield-Stress Liquids", Applied Rheology, vol. 14, pp. 296-302.

[4] De Souza Mendes, P.R., 2007. "Dimensionless non-Newtonian fluid mechanics", Journal of Non-Newtonian Fluid Mechanics, vol. 147, pp. 109-116.

[5] De Souza Mendes, P.R., Naccache, M.F., Varges, P.R., Marchesini, F.H., 2007. "Flow of viscoplastic liquids through axisymmetric expansions-contractions", Journal of NonNewtonian Fluid Mechanics, vol. 142, pp. 207-217.

[6] De Souza Mendes, P.R., 2009. "Modeling the thixotropic behavior of structured fluids", Journal of Non-Newtonian Fluid Mechanics, vol. 164, pp. 66-75.

[7] De Souza Mendes, P.R., 2011. "Thixotropic elasto-viscoplastic model for structured fluids", Journal of Non-Newtonian Fluid Mechanics, vol. 7, pp. 2471-2483.

[8] De Souza Mendes, P.R., Thompson, R. L. 2012. "A critical overview of elasto-viscoplastic thixotropic modeling", Journal of Non-Newtonian Fluid Mechanics, vol. 187-188, pp. $8-15$.

[9] Franca, L.P., Frey, S.L., 1992. "Stabilized finite element methods: II. The incompressible Navier-Stokes equations", Computer Methods in Applied Mechanics and Engineering, vol. 99, pp. 209-233. 